\title{
Microbiological efficacy of early MRSA treatment in cystic fibrosis in a randomised controlled trial
}

\author{
Marianne Sponer Muhlebach, ${ }^{1}$ Valeria Beckett, ${ }^{2}$ Elena Popowitch, ${ }^{3}$ Melissa B Miller, ${ }^{3}$ \\ Arthur Baines, ${ }^{2}$ Nicole Mayer-Hamblett, ${ }^{2,4}$ Edith T Zemanick, ${ }^{5}$ Wynton C Hoover, ${ }^{6}$ \\ Jill M VanDalfsen, ${ }^{2}$ Preston Campbell, ${ }^{7}$ Christopher H Goss, ${ }^{2,4,8}$ STAR-too study team
}

- Additional material is published online only. To view please visit the journal online (http://dx.doi.org/10.1136/ thoraxjnl-2016-208949).

For numbered affiliations see end of article.

\section{Correspondence to} Dr Marianne S Muhlebach, Department of Pediatrics, University of North Carolina, 450 MacNider CB 7217, Chapel Hill, NC 27599, USA; Marianne_Muhlebach@med. unc.edu

Received 22 May 2016 Revised 30 September 2016 Accepted 12 October 2016 Published Online First 15 November 2016

\section{SLinked}

- http://dx.doi.org/10.1136/ thoraxjnl-2016-209605

CrossMark

$$
\begin{aligned}
& \text { To cite: Muhlebach MS, } \\
& \text { Beckett V, Popowitch E, } \\
& \text { et al. Thorax 2017;72: } \\
& \text { 318-326. }
\end{aligned}
$$

\section{ABSTRACT}

Objective To evaluate microbiological effectiveness, that is, culture negativity of a non-blinded eradication protocol $(\mathrm{Rx})$ compared with observation (Obs) in clinically stable cystic fibrosis participants with newly positive methicillin resistant Staphylococcus aureus (MRSA) cultures.

Design This non-blinded trial randomised participants ages $4-45$ years with first or early ( $\leq 2$ positive cultures within 3 years) MRSA-positive culture without MRSAactive antibiotics within 4 weeks $1: 1$ to Rx or Obs. The Rx protocol was: oral trimethoprim-sulfamethoxazole or if sulfa-allergic, minocycline plus oral rifampin; chlorhexidine mouthwash for 2 weeks; nasal mupirocin and chlorhexidine body wipes for 5 days and environmental decontamination for 21 days. The primary end point was MRSA culture status at day 28.

Results Between 1 April 2011 to September 2014, 45 participants (44\% female, mean age 11.5 years) were randomised (24 Rx, 21 Obs). At day 28, 82\% ( $n=18 / 22)$ of participants in the Rx arm compared with $26 \%(n=5 /$ 19 ) in the Obs arm were MRSA-negative. Adjusted for interim monitoring, this difference was $52 \%(95 \% \mathrm{Cl}$ $23 \%$ to $80 \%, p<0.001)$. Limiting analyses to participants who were MRSA-positive at the screening visit, $67 \%(8 / 12)$ in the Rx arm and 13\% (2/15) in the Obs arm were MRSA-negative at day 28 , adjusted difference: $49 \%$ (95\% Cl 22\% to $71 \%$, $p<0.001)$. Fifty-four per cent in the Rx arm compared with 10\% participants in the Obs arm remained MRSA-negative through day 84 . Mild gastrointestinal side effects were higher in the Rx arm.

Conclusions This MRSA eradication protocol for newly acquired MRSA demonstrated microbiological efficacy with a large treatment effect.

Trial registration number NCT01349192.

\section{INTRODUCTION}

Infection with methicillin-resistant Staphylococcus aureus (MRSA) continues to have a significant impact in hospital and community acquired infections. ${ }^{1}{ }^{2}$ In subjects with cystic fibrosis (CF) the prevalence of MRSA-positive respiratory cultures increased from $11.9 \%$ in 2003 to $25.6 \%$ in 2013 in US CF-centres and contributes to adverse outcomes in $\mathrm{CF}^{3}{ }^{4}$ Cross-sectional, epidemiological studies demonstrated that MRSA was associated with lower lung function in $\mathrm{CF}^{5} 6$ and greater use of medical therapies. ${ }^{7}$ Longitudinal outcomes of

\section{Key messages}

What is the key question?

- Is aggressive treatment of incident methicillin-resistant Staphylococcus aureus (MRSA)-positive respiratory culture in cystic fibrosis effective at reducing MRSA culture positivity and is it clinically safe?

What is the bottom line?

- This multifaceted oral, topical and environmental treatment protocol demonstrated a strong microbiological treatment effect in children and adults with few treatment related side effects, which were mostly gastrointestinal and skin related.

\section{Why read on?}

- To learn about the duration of MRSA-negative cultures and secondary outcomes, that showed favourable trends despite a small sample size.

MRSA in CF have noted differing results; one study found no difference in lung function decline whereas another study showed greater lung function decline in those acquiring persistent MRSA infection. $^{8} 9$ Similarly, patients with CF with persistent MRSA infection may have increased mortality. ${ }^{10}$

Treatment approaches highlighted by case series have varied widely from observation to long-term or intravenous antibiotics with the goal of eradicating MRSA. ${ }^{11-13}$ Despite the evolving concern of this organism in the CF community, to date there are no randomised studies demonstrating if treating MRSA at initial detection can eradicate MRSA and prevent chronic respiratory infection. Despite MRSA rates being significantly lower than in the USA, many European countries treat any positive respiratory cultures of MRSA and Methicillin susceptible Staphylococcus aureus (MSSA); alternatively, US guidelines do not recommend treatment of incident MRSA or MSSA in $\mathrm{CF}^{5}$ The risks of indiscriminate and prolonged treatment of MRSA acquisition include the emergence of new/increasingly resistant organisms and treatment related toxicity.

The current study aimed to evaluate the 28-day safety and microbiological efficacy, that is, microbiological treatment effect, of an MRSA eradication 
protocol in patients with CF with newly acquired MRSA as compared with observation alone. We hypothesised that patients with CF who are clinically stable at time of first detection of MRSA in a respiratory culture are more likely to be MRSA culture-negative following an intense multifaceted eradication protocol compared with the current US standard of care of not treating MRSA when patients are stable.

\section{MATERIALS AND METHODS \\ Study centres}

The trial was conducted from 1 April 2011 to September 2014 at $14 \mathrm{CF}$ foundation accredited care centres in the USA. The trial was coordinated by the CF Foundation Therapeutics Development Network Coordinating Center (Seattle, Washington, USA) and registered on clinicaltrials.gov (NCT01349192).

\section{Study participants}

Eligibility criteria included a confirmed diagnosis of CF, age 4-45 years at time of consent and a new onset MRSA-positive culture from sputum, or oropharyngeal (OP) swab or bronchoscopy. New onset was defined as an MRSA-positive culture within 6 months that was either the first lifetime MRSA-positive culture or new emergence of MRSA after at least 1 year of documented negative cultures (minimum of two cultures/year while off MRSA active antibiotics) for MRSA in participants with up to two MRSA-positive cultures in the past 3.5 years. After the planned interim review the Data Monitoring Committee (DMC) recommended that the number of study sites be increased and that subjects MRSA-negative at screening visit be included if the initial clinical MRSA isolate was available. This was to enhance enrolment and to reflect clinical practice. Participants had to be clinically stable within the 14 days prior to screening. Exclusion criteria included having received antibiotics with activity against MRSA or use of an investigational drug within 28 days of screening, and $\mathrm{FEV}_{1}<30 \%$ of predicted based on reference equations. ${ }^{14} 15$ Participants with contraindications for study medications, that is, allergy or renal or hepatic dysfunction were not eligible. Microbiological contraindications were resistance of the available MRSA isolate to trimethoprimsulfamethoxazole (TMP-SMX) and minocycline or to rifampin.

\section{Randomisation and blinding}

Participants were randomised (1:1) to an MRSA eradication protocol as outlined below or to no treatment within strata defined by site, age (4-12 years, 13-45 years) and presence of Pseudomonas aeruginosa at screening. Randomisation assignments were generated via a centralised, secure web based randomisation system for each enrolled subject. Study personnel and participants were not blinded to the treatment regimen.

\section{Treatment regimen $(\mathbf{R x})$}

The treatment protocol for those randomised to the MRSA eradication protocol consisted of two oral antibiotics for 2 weeks combined with nasal, skin and oral decontamination as well as 3 weeks enhanced household cleaning. Study medications were oral TMP-SMX dosed as per CF guidelines at $8 \mathrm{mg} / \mathrm{kg} \mathrm{TMP} / 40 \mathrm{mg} / \mathrm{kg} \mathrm{SMX}$ for children $<40 \mathrm{~kg}$ and $320 \mathrm{mg} / 1600 \mathrm{mg}$ for adults, given twice daily for 14 days. In participants intolerant to TMP-SMX, minocycline, if $>8$ years, at a dose of $100 \mathrm{mg}$ twice daily was substituted. All participants randomised to the treatment protocol received combination therapy with rifampin $(15 \mathrm{mg} / \mathrm{kg} /$ day up to $40 \mathrm{~kg}$ or $300 \mathrm{mg}$ twice daily). Nasal mupirocin and whole body cleansing with chlorhexidine wipes were used for 5 days in addition to twice daily gargling with $0.12 \%$ chlorhexidine gluconate oral rinse for 14 days. Enhanced household cleaning included weekly washing of linens and towels, wiping down high contact surfaces, for example, toys and computers with chlorhexidine, and extra cleaning of airway clearance devices. Selection of the $\mathrm{Rx}$ regimen was based on decolonisation strategies in non-CF populations and TMP-SMX was selected based on a US observational trial of CF which had shown that most MRSA isolates were susceptible to TMP-SMX and rifampin, with very low resistance rates to mupirocin. ${ }^{1617}$

For participants randomised to the observation (Obs) arm, treatment with anti-MRSA therapy prior to day 28 was only allowed for a protocol defined exacerbation with choice of antibiotics as per the participant's treating physician. Use of anti-MRSA antibiotics after day 28 was allowed for both arms.

\section{Clinical evaluations}

Medical history, physical examination, specimen sampling for microbiology (OP, nasal, groin and axilla swabs on all participants, additional sputum in those expectorating) and spirometry were obtained at the screening visit (day 14). Clinical evaluations, physical examination and spirometry were performed on day 1 (randomisation), day 15, day 28, day 84 and day 168 . Follow-up after day 28 was used to assess durability of treatment effect. Pulmonary function testing was performed in accordance with American Thoracic Society standards. ${ }^{18}$ Adverse events and concomitant medications were recorded during each visit and by phone calls conducted on day 7 . Protocol defined pulmonary exacerbations were defined using a combination of spirometry, X-ray and clinical symptoms as in prior studies. $^{19}$

\section{Primary and secondary outcomes}

The primary outcome of the study was difference in the proportion of MRSA-negative subjects based on OP-swab or sputum on day 28 between the two study arms. Secondary end points included safety, tolerability of the treatment regimen, protocol adherence, duration of microbiological effect, number of pulmonary exacerbations, use of antibiotics, change in spirometry (as measured by $\mathrm{FEV}_{1}$ ), respiratory symptoms as measured by the CF-specific patient outcomes: Cystic Fibrosis Questionnaire Revised respiratory domain scores and Cystic Fibrosis Respiratory Symptom Diary Chronic Respiratory Infection Symptom Scale, and weight.

\section{Statistical analysis}

The study design specified randomisation of 90 participants providing $80 \%$ power to detect a difference of $30 \%$ or greater in the proportion of respiratory cultures negative for MRSA at day 28 ( $\mathrm{Rx}$ minus Obs). The sample size calculations assumed a dropout rate of $10 \%$, ensuring 40 randomised participants to $\mathrm{Rx}$ and 40 to Obs arms.

Safety outcomes were monitored throughout the trial by a DMC appointed by the CF Foundation Safety Monitoring Board. One interim analysis with early stopping for futility was scheduled to take place after approximately half of the participants had been randomised into the study and had completed the day 28 visit. Because of slow accrual, an early interim review and futility analysis of the primary end point was initiated when 24 participants had completed the first 28 days of the study. Upon review of the interim results, the DMC recommended continuance of the study with a second interim analysis for early efficacy. The study team remained blinded to findings presented to the DMC. Statistical ramifications of this 


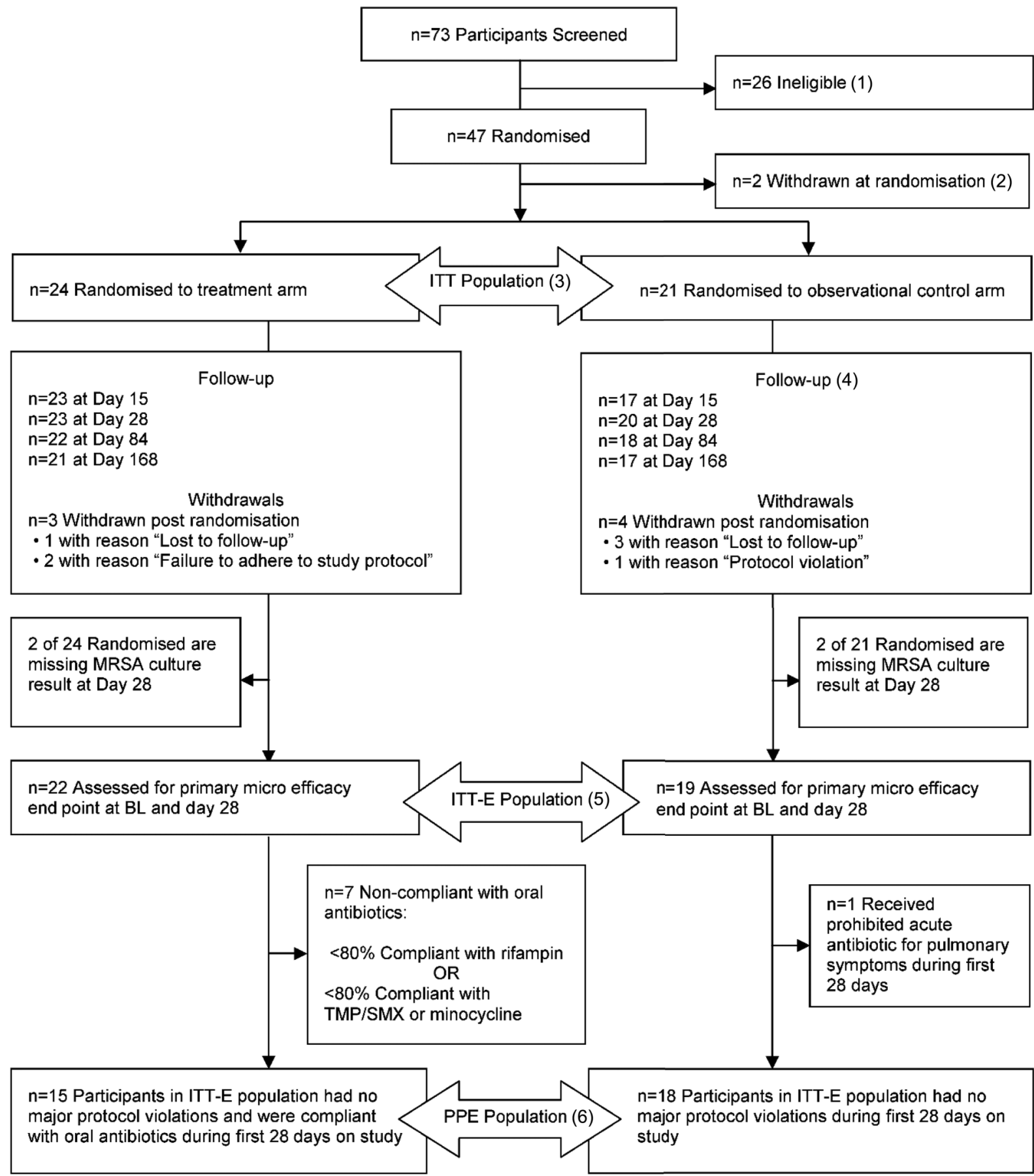

Figure 1 CONSORT diagram of participant disposition. Flow diagram of participants through each stage of the randomised trial. (1) Nine participants did not meet the inclusion criteria of being clinically stable. One of these participants was subsequently rescreened but failed the inclusion criteria of methicillin-resistant Staphylococcus aureus (MRSA)-positive culture at screening or within 6 months prior to screening. Eleven participants failed the inclusion criteria of MRSA-positive culture at screening or within 6 months prior to screening. One participant did not meet the inclusion criteria by withdrawing their consent. One participant did not meet the inclusion criteria of having a documented cystic fibrosis (CF) diagnosis. Two participants met the exclusion criteria of receiving anti-MRSA antibiotics within 28 days prior to screening. One participant met the exclusion criteria of abnormal renal function at screening. One participant met the exclusion criteria that warranted a screen failure due to investigator's opinion. (2) Two participants randomised to the observational control arm withdrew with reason 'Subject decision'. (3) The intent-to-treat (ITT) population is defined as participants who are randomised to a study arm and followed post randomisation. The ITT population is used for all safety and secondary efficacy analyses. (4) One site, which enrolled a total of four participants (two in the treatment arm, two in the observational control arm), experienced numerous study conduct issues and protocol violations resulting in missing primary end point and other end point data. One of these four participants had withdrawn immediately following randomisation to the observational control arm. The remaining three participants' data are summarised as available post randomisation. (5) The ITT-efficacy (ITT-E) population consists of all the participants in the ITT population who were assessed for the primary microbiological efficacy end point at baseline and day 28. The ITT-E population is used for the primary efficacy analyses. (6) The per-protocol-efficacy (PPE) population is comprised of all participants in the ITT-E population excluding the participants with major protocol violation or those non-compliant with oral antibiotic use during the first 28 days of the study. The PP population is used in sensitivity analyses of primary efficacy end point. TMP-SMX, trimethoprim-sulfamethoxazole. BL, baseline; PP, is per protocol. 
unplanned efficacy analysis are addressed in the online supplementary material.

All of the safety and secondary efficacy analyses were conducted on the intent-to-treat (ITT) population. The primary efficacy analyses were performed on the ITT-efficacy (ITT-E) population. The per-protocol-efficacy (PPE) population was used in sensitivity analyses of the primary end point. The detailed definitions of analyses populations are provided in figure 1. T-tests were used to compare continuous variables by study arm. The comparisons of proportions were performed using Fisher's exact test, with corresponding 95\% CI derived using the Newcombe-Wilson method. Event rate comparisons were performed using Poisson regression. In the analyses of the primary end point, the difference in the proportion of respiratory cultures negative for MRSA at day 28 (Rx minus Obs), the treatment effect, 95\% CIs and p value estimates were adjusted for the interim reviews. The use of group sequential stopping rules alters the sampling distribution of the usual fixed sample statistics and so adjustments need to be made to compute the point estimates, CIs and $\mathrm{p}$ values. The results of final analyses had to be adjusted for bias to account for multiple looks at the data during the two interim reviews (referred to as 'adj. for interim monitoring').

$\mathrm{p}$ Values and CIs are two-sided with 0.05 significance level; analyses were performed using SAS (V.9.4, SAS Institute, Cary, North Carolina, USA, 2013) and R (V.3.2.1, The R Foundation for Statistical Computing, Vienna, Austria, 2015).

\section{RESULTS}

Although the planned sample size was 90 patients, the DMC recommended that the enrolment of new participants be stopped early with ongoing follow-up of enrolled subjects, after interim review by the DMC showed a statistically significant microbiological treatment effect. At the time the trial was stopped, 73 participants had been screened at 14 participating centres; 45/73 participants were randomised and followed postrandomisation (ITT population), 24 in the $\mathrm{Rx}$ and 21 in the Obs arms (figure 1).

Clinical characteristics of the ITT participants $(n=45)$ showed a mean age of 11.5 years with well-preserved lung function (table 1). The two arms were comparable at baseline (table 1) and distribution of participants who had a sputum sample in addition to OP swab did not differ by study arm (no participant included based on bronchoscopy results). Of the 45 randomised participants, 41 (22 Rx and 19 Obs) were included in the ITT-E analyses. Although the protocol allowed changing from TMP-SMX to minocycline in case of side effects, none changed treatment during the study; however, two subjects in the Rx arm were started on minocycline due to previously known intolerances to TMP-SMX. A total of seven participants withdrew post randomisation (three (13\%) in $\mathrm{Rx}$ and four (19\%) in Obs); four withdrew before day 28 visit and were not assessed for the primary microbiological end point (figure 1).

Table 2 summarises the primary end point, that is, changes in MRSA culture status from screening through day 28. Those who were not positive at screening had a confirmed MRSA isolate at a clinic visit within a median of 46 days (range 14154 days) prior to screening. The proportion of participants in the ITT-E population who were MRSA-negative at day 28 was $82 \%(n=18 / 22)$ in the $\mathrm{Rx}$ arm compared with $26 \%(\mathrm{n}=5 / 19)$ in the Obs arm. Adjusted for the interim reviews, the difference in the proportion being MRSA-negative at day 28 (Rx minus Obs) was $52 \%$ (95\% CI $23 \%$ to $80 \%, \mathrm{p}<0.001)$. In a sensitivity analysis limited to participants who were
Table 1 Demographics and baseline characteristics by study arm

\begin{tabular}{|c|c|c|c|}
\hline & \multicolumn{2}{|l|}{ No. (\%) } & \multirow[b]{2}{*}{$\begin{array}{l}\text { Total } \\
(n=45)\end{array}$} \\
\hline & $\begin{array}{l}\text { Treatment } \\
(n=24)\end{array}$ & $\begin{array}{l}\text { Observational } \\
\text { control } \\
(n=21)\end{array}$ & \\
\hline Sex-female & $10(42)$ & $10(48)$ & $20(44)$ \\
\hline \multicolumn{4}{|l|}{ Race } \\
\hline Caucasian & $19(79)$ & $17(81)$ & $36(80)$ \\
\hline Hispanic & $3(13)$ & $2(10)$ & $5(11)$ \\
\hline African-American & $1(4)$ & $1(5)$ & $2(4)$ \\
\hline Other & $1(4)$ & $1(5)$ & $2(4)$ \\
\hline \multicolumn{4}{|l|}{ Genotype } \\
\hline F508 del homozygous & $6(25)$ & $12(57)$ & $18(40)$ \\
\hline F508 del heterozygous & $14(58)$ & $7(33)$ & $21(47)$ \\
\hline Other* & $4(17)$ & $2(10)$ & $6(13)$ \\
\hline \multicolumn{4}{|l|}{ Age group (years) } \\
\hline $4-12$ & $13(54)$ & $15(71)$ & $28(62)$ \\
\hline$>12-18$ & $6(25)$ & $5(24)$ & $11(24)$ \\
\hline$>18$ & $5(21)$ & $1(5)$ & $6(13)$ \\
\hline $\begin{array}{l}\text { Pseudomonas } \\
\text { aeruginosa-positive }\end{array}$ & $4(17)$ & $4(19)$ & $8(18)$ \\
\hline \multicolumn{4}{|l|}{$\mathrm{FEV}_{1} \%$ predicted group $\dagger$} \\
\hline $30-50 \%$ predicted & 1 (5) & $0(0)$ & $1(3)$ \\
\hline$>50-75 \%$ predicted & 1 (5) & $0(0)$ & $1(3)$ \\
\hline$>75-100 \%$ predicted & 7 (35) & $5(29)$ & $12(32)$ \\
\hline \multirow[t]{2}{*}{$>100 \%$ predicted } & $11(55)$ & $12(71)$ & $23(62)$ \\
\hline & \multicolumn{3}{|l|}{ Mean (SD) } \\
\hline Age (years) & $12.3(6.6)$ & $10.5(5.5)$ & $11.5(6.1)$ \\
\hline $\mathrm{FEV}_{1} \%$ predictedt & $98.5(21.6)$ & $101.2(11.8)$ & $99.8(17.6)$ \\
\hline Weight (kg) & $40.5(17.0)$ & $38.2(19.8)$ & $39.4(18.2)$ \\
\hline Weight (\%)‡ & $50.5(27.4)$ & $53.7(23.9)$ & $52.0(25.5)$ \\
\hline Body mass index (\%)‡ & $60.8(25.4)$ & $64.6(20.5)$ & $62.7(23.0)$ \\
\hline
\end{tabular}

This table summarises demographic and baseline characteristics by study arm in the ITT population. All measures were recorded at screening.

* Other refers to participants with either two known, non-delta F508 CF mutations, or one known, non-F508 del CF mutation and one unidentified allele which has not been classified as a CF mutation.

FFor participants 6 years or older, $\mathrm{FEV}_{1} \%$ predicted is calculated based on the Wang (boys $<18$ years, girls $<16$ years) or Hankinson (boys $\geq 18$ years, girls $\geq 16$ years) reference equations. Percentages are based on number of participants with FEV measurements available (20 in the treatment arm and 17 in the observational control arm).

$\ddagger$ The centiles are derived using CDC standards for participants $\leq 20$ years old. $\mathrm{CF}$, cystic fibrosis; ITT, intent-to-treat.

MRSA-positive at the screening visit, $67 \%$ in the $\mathrm{Rx}$ compared with $13 \%$ in the Obs arm were MRSA-negative at day 28, with an adjusted difference of $49 \%$ (95\% CI $22 \%$ to $71 \%$, $\mathrm{p}<0.001)$. These results prompted the DMC to recommend early study closure (see online supplementary material labelled Statistical Monitoring Guidelines and Interim Primary End point Analyses).

Online supplementary table S1 shows results of sensitivity analyses within the first 28 days for the primary efficacy end point. These sensitivity analyses addressed the use of anti-MRSA antibiotics within the first 28 days. For all of the sensitivity analyses, the inference was consistent with the primary analysis and in particular, the observed treatment effect was stronger in the PPE population.

In the ITT population, 15 participants $(65 \%)$ were compliant in their use of both oral antibiotics by taking at least $80 \%$ doses of rifampin and TMP-SMX, or minocycline (only two subjects in this group were treated with minocycline). Overall, the 
Table 2 Microbiological effect at day 28

\begin{tabular}{|c|c|c|c|c|}
\hline & $\begin{array}{l}\text { Treatment } \\
(\mathrm{N}=24)\end{array}$ & $\begin{array}{l}\text { Observational } \\
\text { control } \\
(\mathrm{N}=21)\end{array}$ & $\begin{array}{l}\text { Difference } \\
(95 \% \mathrm{Cl})\end{array}$ & p Value \\
\hline \multicolumn{5}{|l|}{ Screening } \\
\hline Number screened & 24 & 21 & & \\
\hline MRSA-positive at screen, $\mathrm{n}(\%)$ & $14(58 \%)$ & $17(81 \%)$ & $-23 \%(-45 \% \text { to } 4 \%)^{*}$ & $0.12 \dagger$ \\
\hline \multicolumn{5}{|l|}{ Day 28} \\
\hline Number completed & 22 & 19 & & \\
\hline MRSA-negative at day $28, \mathrm{n}(\%)$ & $18(82 \%)$ & $5(26 \%)$ & $52 \%(23 \%$ to $80 \%) \ddagger$ & $<0.001 \ddagger$ \\
\hline \multicolumn{5}{|l|}{ Change from screening to day 28} \\
\hline Number MRSA-positive cultures at screening§ & 12 & 15 & & \\
\hline $\begin{array}{l}\text { Changed to MRSA-negative from } \\
\text { screening to day } 28, \mathrm{n}(\%)\end{array}$ & $8(67 \%)$ & $2(13 \%)$ & $49 \%(22 \%$ to $71 \%) \ddagger$ & $<0.001 \ddagger$ \\
\hline \multicolumn{5}{|c|}{ 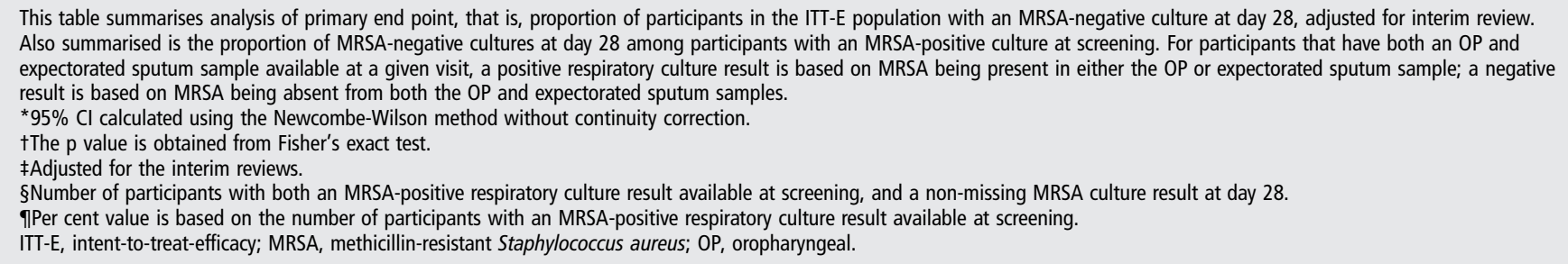 } \\
\hline
\end{tabular}

protocol was acceptable to patients and families with a relatively low treatment burden (see online supplementary material). Adherence with environmental decontamination was very good with two participants (9\%) reporting missing $\geq 5$ days of wipes and one participant missing $\geq 1$ time of washing the linens. There were three instances of oral antibiotic discontinuation due to adverse events 'probably related' to study drug: two were temporary discontinuation of rifampin due to gastroinstestinal (GI) complaints, whereas one participant had to discontinue all antibiotics due to urticaria. None of the adverse events was considered serious or required hospitalisation. Unrelated to adverse events one participant discontinued rifampin early and one participant reported taking TMP/SMX only once daily.

Two serious adverse events occurred during the first 28 days on study, one in the Rx arm (increased cough) and one in the Obs arm (cellulitis of the eyelid). Types of adverse events were more likely related to gastrointestinal and skin/subcutaneous tissue disorders in the treatment arm. No significant laboratory related adverse events were identified (see online supplementary table S2 and Safety Laboratory Assessment). There were no microbiological adverse events (ie, no emergent MRSA resistances to antibiotics used or appearance of small colony variants).

Use of anti-MRSA antibiotics is shown by individual participants in figure 2 from screening through day 168 by study arm together with MRSA culture status. After day 28 , nine $(38 \%)$ participants in the $\mathrm{Rx}$ and nine (43\%) in the Obs arm were treated with anti-MRSA antibiotics. The usage of non-MRSA active acute oral, inhaled or intravenous antibiotic was comparable between treatment arms during the first 28 days as well as throughout the study. Participant-specific MRSA culture results show that 13 of 24 participants (54\%) in the $\mathrm{Rx}$ arm were MRSA-negative at day 28 and remained negative through day 84 (figure $2 \mathrm{~A}$ ) as compared with two of 21 (10\%) participants in the Obs arm (figure 2B). The impact of acute antibiotic administration in the Obs arm on the treatment effect is examined in online supplementary tables S3.1 and S3.2.

Two participants, one in each arm, were hospitalised during the first 28 days of the study. Over the entire course of the study, two $(8 \%)$ participants in the $\mathrm{Rx}$ arm were hospitalised 3 times and five (24\%) were hospitalised in the Obs arm 11 times (difference $=-15 \%, 95 \% \mathrm{CI}-38 \%$ to $6 \%, \mathrm{p}=0.22$ ). The rate of hospitalisation from screening through day 168 was significantly lower in the $\mathrm{Rx}$ arm versus the Obs arm $(\mathrm{RR}=0.22$, 95\% CI 0.05 to $0.72, \mathrm{p}=0.01$ ).

The proportion of participants experiencing at least one pulmonary exacerbation between screening and day 28 (calculated as the proportion of patients experiencing an event per 28 days of follow-up) was $13 \%$ in the $\mathrm{Rx}$ arm as compared with $33 \%$ in the Obs arm (95\% CI $-44 \%$ to $4 \%, \mathrm{p}=0.15)$. Similarly, the rate of exacerbation from screening through day 28 was lower in the $\mathrm{Rx}$ arm versus the Obs arm, $(\mathrm{RR}=0.36,95 \%$ CI 0.08 to 1.29 , $\mathrm{p}=0.12)$, although not statistically significant.

At screening, 14 of 45 participants had nasal MRSA colonisation. The proportion colonised was similar in the two treatment arms: 6 of $24(25 \%)$ in the $\mathrm{Rx}$ and 8 of $21(38 \%)$ in the Obs $(\mathrm{p}=0.52)$ arms. No treatment related differences emerged. Only one patient was MRSA colonised at the skin (see online supplementary tables S4.1 and S4.2). Participants with persistent or re-emergent MRSA infection kept the same Staphylococcal chromosome cassette mec (SCCmec) type. There were no differences in proportion colonised with $P$. aeruginosa at screening, with 4 of $24(17 \%)$ in the Rx and 4 of $21(19 \%)$ in the Obs arms ( $p>0.999$ ). No differences emerged during the course of the trial.

At day 28, the mean relative change in $\mathrm{FEV}_{1}$ (litres) from screening was $2.5 \%$ in the $\mathrm{Rx}$ arm $(\mathrm{n}=19)$ and $-2.4 \%$ in the Obs arm $(n=16)($ difference $=4.9 \%, 95 \%$ CI $-0.6 \%$ to $10.4 \%$, $\mathrm{p}=0.08$ ); the mean absolute change in $\mathrm{FEV}_{1}$ (\% predicted) was $0.7 \%$ in the $\mathrm{Rx}$ arm and $-4.1 \%$ in the Obs arm with a difference of $4.8 \%$, (95\% CI $-0.9 \%$ to $10.5 \%, p=0.10)$. At day 168 , the differences $(\mathrm{Rx}-\mathrm{Obs})$ in mean relative change in $\mathrm{FEV}_{1}$ (litres) was $3.1 \%$ (95\% CI $-2.5 \%$ to $8.6 \%, \mathrm{p}=0.27)$ and the mean absolute change in $\mathrm{FEV}_{1}$ (\% predicted), 4.7\% (95\% CI $-0.4 \%$ to $9.8 \%, p=0.07$ ) (see figure 3 ). Online supplementary figures $\mathrm{S} 1.1$ and $\mathrm{S} 1.2$ show the changes in $\mathrm{FEV}_{1}$ (litres) and $\mathrm{FEV}_{1}$ (\% predicted)).

There were no significant differences between study arms with respect to changes in weight or patient reported outcomes (see online supplementary figures S2.1, S2.2, S2.3, S3.1 and S3.2). 
A

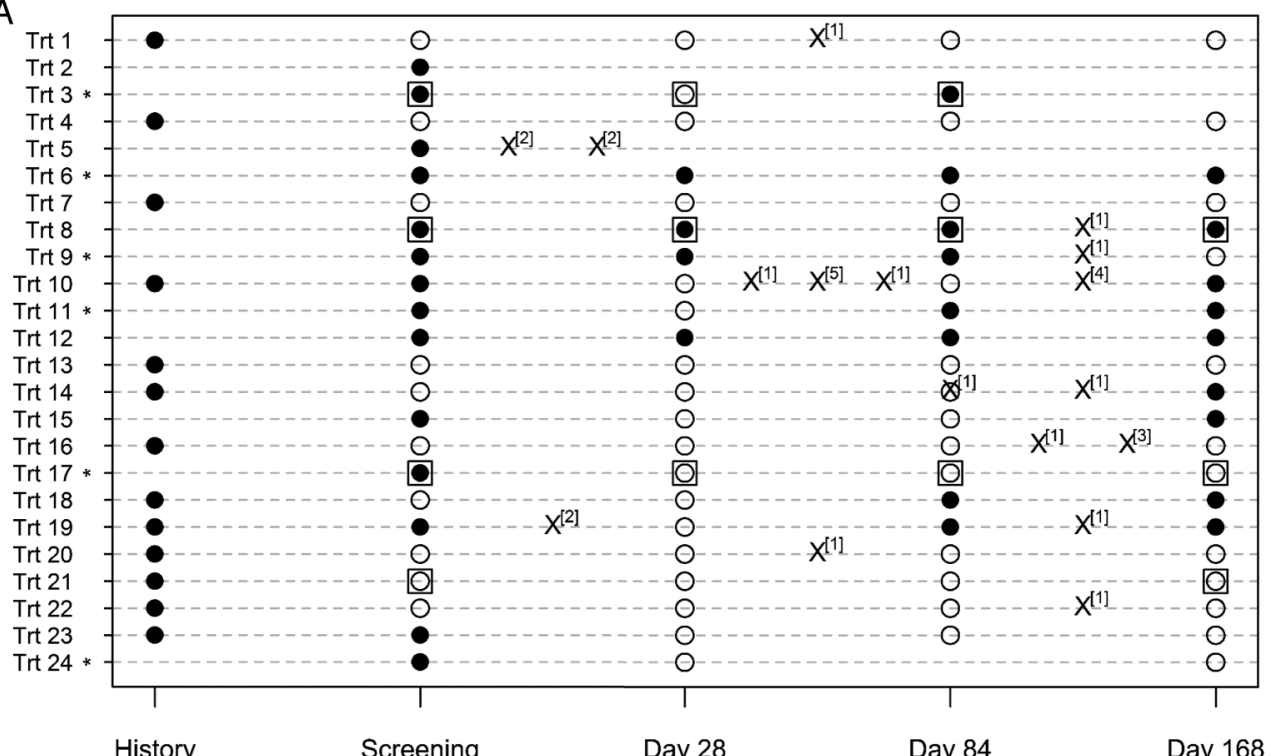

History

Screening

Day 28

Day 84

Day 168

- MRSA-

[1] TMP/SMX

- MRSA+

2] CLINDAMYCIN

[4] LINEZOLID

口 $\mathrm{Pa}+$

[3] DOXYCYCLINE

[5] RIFAMPIN

$x$ Initiated antibiotics

* enrolled pre-amendment

B

- MRSA-

[1] TMP/SMX

- MRSA+

[2] DOXYCYCLINE

[3] RIFAMPIN

- $\mathrm{Pa}+$

$\times$ Initiated antibiotics

* enrolled pre-amendment

Figure 2 (A Rx arm and B Obs arm) Participant-specific methicillin-resistant Staphylococcus aureus (MRSA) culture results through day 168 by treatment arm. Individual participant MRSA culture status across time is shown. History of MRSA-positive isolate is also shown. Participants with who are Pseudomonas aeruginosa-positive $(\mathrm{Pa}+)$ are indicated by $\square$. Acute events treated with anti-MRSA active antibiotics are marked with an ' $X$ '. The locations of the ' $X$ 's indicate the timing of the antibiotic course in relationship to the study visits but do not represent an actual day as measured from screening. Participants enrolled prior to protocol amendment are marked with an asterisk (see Discussion). TMP-SMX,

trimethoprim-sulfamethoxazole.

\section{DISCUSSION}

Prevalence of MRSA in CF is $\sim 26 \%$ in the USA and chronic infection has been associated with higher rates of lung function decline and mortality. ${ }^{9} 10$ The current randomised controlled trial evaluated a comprehensive protocol with two oral antibiotics for 2 weeks combined with nasal, skin and oral decontamination as well as a 3-week environmental decontamination in clinically stable patients with CF. This treatment led to a marked reduction in culture positivity (OP swab or sputum) at 28 days compared with the observational arm. The treatment 
Figure 3 Relative change from screening in $\mathrm{FEV}_{1}$ (litres) over time by study arm (intent-to-treat (ITT) population). Relative change in $\mathrm{FEV}_{1}$ (litres) from baseline to each postbaseline visit for both study arms in the ITT population is shown. At each time point $95 \%$ Cls (using t-distribution approximation) are included. The number of participants at each time point is included in a legend below the figure. Per protocol, participants younger than 6 years of age from both the treatment arm $(n=3)$ and observational control arm $(n=4)$ were not assessed for pulmonary function.

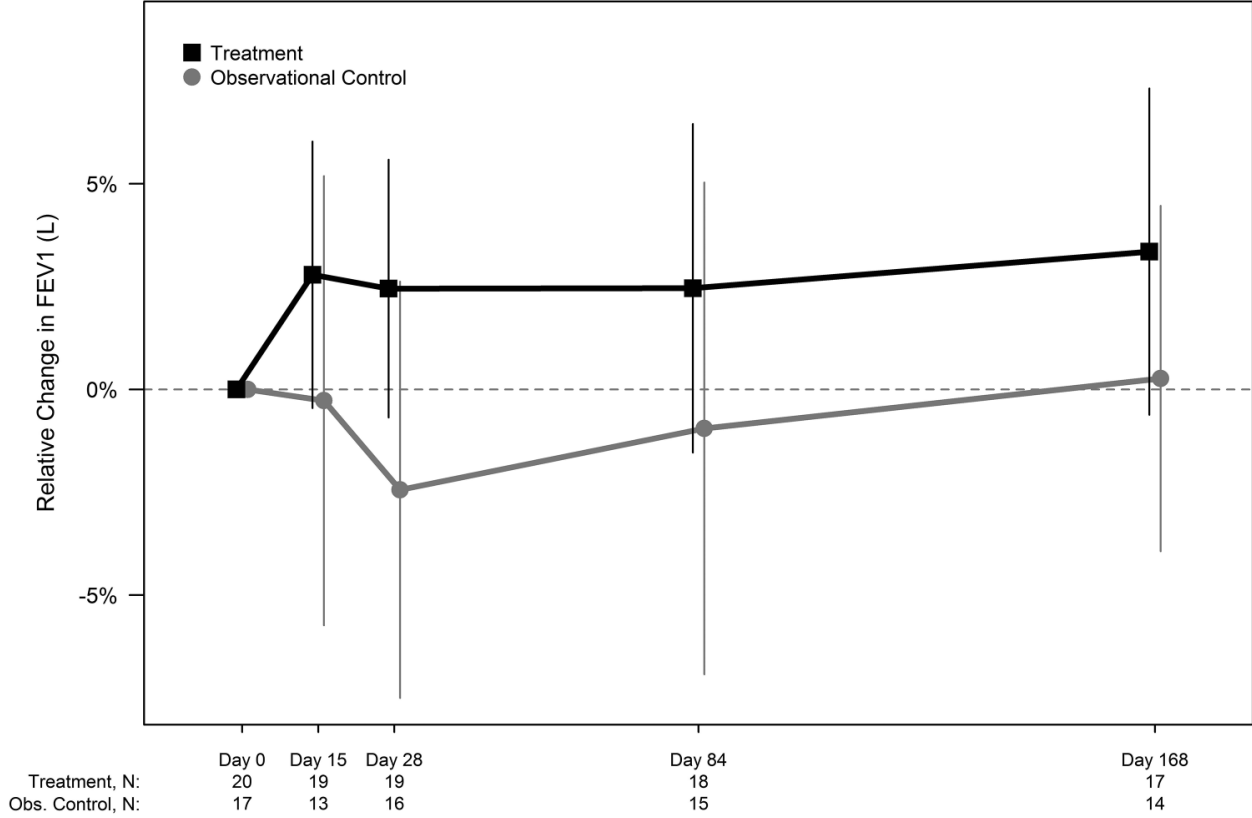

$\begin{array}{rlll}\text { Treatment, N: } & 20 & 19 & 19 \\ \text { Obs. Control, N: } & 17 & 13 & 16\end{array}$ effect was large enough to recommend early study termination by the DMC due to evidence of microbiological efficacy, that is, the high rate or MRSA-negative cultures in the Rx compared with the Obs arm. Positive trends were also seen for secondary outcomes with reduction in the rate of pulmonary exacerbation and a trend towards improved lung function despite the study population having preserved lung function at baseline. As expected for these antibiotics, the rate of GI side effects was higher in the $\mathrm{Rx}$ arm, however the only permanent discontinuation of antibiotics was for urticaria. Thus, the treatment regimen appeared overall safe and, despite some drug related side effects, well tolerated. Despite most participants reporting that the regimen was acceptable, compliance with all aspects of this regimen was not ideal, which may be related to the multifaceted approach.

The possibility of treating MRSA successfully and achieving eradication even in settings of chronic infection has been reported. ${ }^{11-13}{ }^{20-22}$ None of these studies included a control arm and all had smaller sample sizes than the current study. Despite these limitations, they demonstrated the potential benefit of eradication. Of note, these reports included countries and CF centres with much lower MRSA prevalence than the USA, which would be especially relevant in examining rates of MRSA recurrence. A systemic review for the early treatment of MRSA in CF concluded that there were no randomised trials available to assess early eradication. ${ }^{23}$

The protocol specified antibiotics were based on drug availability, cost, synergy and antibiotic susceptibility in CF MRSA isolates in the USA. ${ }^{16} 17{ }^{24}$ Although fusidic acid showed low rates of resistance for US CF MRSA isolates ${ }^{17}$ and that MRSA eradiation protocols outside the USA reported combination therapy with fusidic acid and rifampin, this drug is not approved in the USA. We included nasal or throat decontamination procedures based on the use of mupirocin in non-CF eradication/ decontamination ${ }^{16}$ and the high rate of positive throat cultures in $\mathrm{CF}^{25}$ Skin decontamination was included based on possible high skin colonisation with SCCmec IV isolates and approaches in non-CF decolonisation. ${ }^{26}$ Interestingly, despite high rates of SCCmec IV MRSA, skin positivity was rare and the topical skin treatment may not be essential in CF. Further trials comparing our intense protocol to less elaborate treatment approaches are required to demonstrate whether skin decontamination is required.

This study was primarily designed as a controlled trial of MRSA treatment with a short controlled microbiology outcome. Thus, longer-term clinical outcomes after day 28 in our trial are harder to interpret given the very high rate of oral, intravenous and inhaled antibiotics (many with anti-MRSA activity) in both study arms, even in subjects not culturing MRSA (figure 2). The participant and treatment heterogeneity after the intervention period limited interpretation of long-term clinical outcomes and we are not able to definitively comment on long-term lower airway eradication. At day 84, a higher proportion of participants in the $\mathrm{Rx}$ arm than in the Obs arm were still MRSA-negative. It is difficult to evaluate if recurrence of MRSA is due to persistence not detected by culture or due to reinfection and, although repeat isolates were of the same SCCmec type, this method has insufficient sensitivity to address high degree genetic relatedness. Further studies will need to address these questions. Prior literature has demonstrated heavy antibiotics use in US MRSA-positive CF populations. ${ }^{27-29}$

Two aspects of the study warrant special attention and should be considered in regard to generalisability of results. First, because of poor enrolment rates, the number of study sites was expanded and participants were allowed to be enrolled if they had MRSA isolated from the respiratory tract (sputum or OP culture) at the most recent clinical care visit if this MRSA isolate was available for susceptibility testing. This amendment mirrored common clinical practice and was similar to the approach taken in the Early Pseudomonas Infection Control trial. ${ }^{19}$ Importantly, when the primary end point was analysed based only on participants who were culture-positive for MRSA at 
screening (27/41 in the ITT-E population), the results were similar.

Second, the study was stopped based on an unplanned efficacy interim analysis. Interestingly, early stopping for microbiological efficacy was also initiated in the early inhaled tobramycin eradication trial. ${ }^{30}$ At the first planned review, the DMC recommended an unplanned interim efficacy analysis. In response to this unplanned review, additional statistical analyses evaluated the sensitivity of the results to variations in stopping rules (see online supplementary table S1). ${ }^{31-35}$ Because the stopping rule for efficacy was not specified in advance, this sensitivity analysis assessed how sensitive the inference (related to the difference in the proportion of MRSA-negative between study arms) was to a myriad of stopping rules that the DMC could have considered. These estimates of treatment differences adjusted for the corresponding group sequential stopping rule are shown in detail in online supplementary table S1.

This trial was not blinded to either staff or participants because several of the interventions, for example, rifampin could not be blinded. However, the primary end point was an objective measure, that is, culture positivity. This design may have led to higher use of antibiotics in the Obs arm after day 28.

There are a number of important limitations to interpreting the results of this clinical trial. First, only participants ages 445 years with early MRSA infection were included. Thus, one cannot infer that this protocol would have a similar treatment effect in patient groups not fulfilling these inclusion criteria. Consistent with the highest incidence of MRSA occurring in mid-childhood in the US CF population, the majority of participants were children with $\mathrm{FEV}_{1}>75 \%$ predicted (table 1) and the majority could not expectorate sputum. Further, patients presenting in the setting of an acute exacerbation who were culture-positive for MRSA or who had received antibiotics effective against MRSA in the prior 28 days were excluded. These participant characteristics and other exclusion criteria limit the extension of these results to the broader CF population infected with MRSA. Last, the study was not designed to assess the long-term impact of MRSA eradication or effect of repeated treatment MRSA eradication courses. Data gathered in the observational extension portion of the study noted that both $\mathrm{Rx}$ and Obs arms received multiple courses of antibiotics with potential MRSA activity.

Our study did demonstrate that spontaneous clearance of MRSA at day 28 does occur at a rate of approximately 13\% in those that were culture-positive at screening and $26 \%$ in the entire observation arm. Data from the US CF Registry data collected from 1996 to 2006 indicate that among patients ages $\geq 6$ years up to $50 \%$ of subjects have only one time positive MRSA cultures or intermittent MRSA infection, however treatment history is missing on these subjects. ${ }^{10}$ Such data however do highlight the value of obtaining repeat cultur prior to eradication attempts if one was to employ such a protocol.

\section{CONCLUSION}

This trial is the first randomised clinical trial to study the microbiological impact of an eradication protocol. While there was a significant difference in microbiological success and evidence towards fewer exacerbations in the $\mathrm{Rx}$ arm in this short trial, more questions remain prior to recommending universal early anti-MRSA therapy. These include optimisation of the treatment regimen that is, are all measures necessary and assessing individuals who either have mild exacerbations or are outside the currently selected age range. The high rate of antibiotic use after the primary end point begs the question if a repeat course is indicated in those who remain culture-positive at the end of the treatment. These questions will need further clinical trials and close observation of clinical practice in all countries. However, we did demonstrate that currently available antibiotics with a well established safety profile are effective in early MRSA infection.

\section{Author affiliations}

${ }^{1}$ Department of Pediatrics, University of North Carolina, Chapel Hill, North Carolina, USA

${ }^{2}$ Seattle Children's Research Institute, Seattle, Washington, USA

${ }^{3}$ Department of Microbiology, University of North Carolina, Chapel Hill, North Carolina, USA

${ }^{4}$ Department of Pediatrics, University of Washington, Seattle, Washington, USA ${ }^{5}$ Department of Pediatrics, University of Colorado School of Medicine, Denver, Colorado, USA

${ }^{6}$ Department of Pediatrics, University of Alabama at Birmingham, Birmingham, Alabama, USA

${ }^{7}$ Cystic Fibrosis Foundation, Bethesda, Maryland, USA

${ }^{8}$ Department of Medicine, University of Washington, Seattle, Washington, USA

Acknowledgements The authors thank the CF participants, families of CF children who participated in the study, and whose dedication to research made the trial possible. The authors also thank Bob Beall, PhD and the Cystic Fibrosis Foundation for supporting this clinical trial.

Collaborators STAR-too study team Investigators: Study Acronym STAR-too: STaph Aureus Resistance - treat or observe. Participating Sites: Site (Investigator and lead Research Coordinator): University of Washington (Christopher H Goss, Debbie Ng); Minneapolis Children's Affiliate (John McNamara, Mahrya Johnson); Texas Children's Hospital (Silby Moonnumakal, Nicoline Schaap); University of Colorado (Edith Zemanick, Meg Anthony); University of Cincinnati (John P Clancy); Seattle Children's Hospital (Ronald Gibson, Sharon McNamara); Washington University, St. Louis Children's (Peter Michelson, Tina Hicks); University of Texas Southwestern (Preeti Sharma, Andrew Hebert); University of Alabama at Birmingham (Wynton Hoover Katie Brand); University of Florida at Gainesville (Pamela Schuler, Dawn Baker); University of Michigan (Amy Filbrun, Marisa Linn); University of Nebraska (Paul Sammut, Raquel Telfer); University of North Carolina at Chapel Hill (Marianne Muhlebach, Kelly Moormann); Ft. Worth Cook Children's Hospital (Karen Schultz, Heather Urbanek). Data and Safety Monitoring Board/DMC: Margaret Guill, MD, DMC Chair; Dartmouth-Hitchcock Medical Center, Pediatric Pulmonary Medicine. John J LiPuma, MD, Pediatric Infectious Disease Medicine, University of Michigan. Marci Sontag, PhD, Epidemiology/Community Health, Preventive Medicine/ Biometrics, The Children's Hospital, Aurora, Colorado. Susan Murray, ScD, University of Michigan, School of Public Health, Department of Biostatistics (CFFT DMC Ad-hoc Specialist, Sequential Monitoring). Miriam Hunt, CFFT DMC, Prog. Coord., Sr. CFF Therapeutics Development Coordinating Center: Dianne L. Howe, Jasna Hocevar-Trnka, Rose Mitchell, Lynette Browne.

Contributors MSM: inception of study, study design, supervising conduction of study, discussion of data and writing, revising manuscript; communication with all authors and Thorax. EP contributed to protocol development; performed all laboratory analyses on bacterial samples. MBM: Contributed to design of the microbiology end points and provided oversight of all MRSA related processing and interpretation of MRSA typing results. PC contributed to the study inception and ongoing advice during the trial. WCH contributed to patient recruitment and enrolment at his study site, provided input for study modification and ongoing feedback on the protocol; participated in review and modifications of the final manuscript. ETZ contributed to patient recruitment and enrolment at her study site, provided input for study modification and ongoing feedback on the protocol; participated in review and modifications of the final manuscript. $\mathrm{CHG}, \mathrm{NM}-\mathrm{H}$ and JMVD contributed to study conception and study design. $\mathrm{NM}-\mathrm{H}, \mathrm{VB}$ and $\mathrm{AB}$ participated in data management and statistical analyses. All authors participated in data analysis/interpretation, drafting and/or revising the manuscript for intellectual content, and editing the manuscript for final approval.

Funding The research for this article was supported by the Cystic Fibrosis Foundation grant number STAR10K, and CTSA at individual sites: UL1TR001111, UL1 TR000433, UL1 TR000077, UL1 TR000423, NCT02249182, UL1 TR001417, UL1 TR000448, UL1 TR001082, UL1 RR025780. CHG receives funding from the Cystic Fibrosis Foundation, the NIH (R01HL103965, R01HL113382, R01Al101307, U M1HL119073, P30DK089507) and the FDA (R01FD003704).

Competing interests None declared.

Patient consent Obtained.

Ethics approval The institutional review board of each study site. 
Provenance and peer review Not commissioned; externally peer reviewed.

\section{REFERENCES}

1 CDC. Active Bacterial Core Surveillance Report, Emerging INfections Program Network, MRSA. Secondary Active Bacterial Core Surveillance Report, Emerging INfections Program Network, MRSA, 2012. http://www.cdc.gov/abcs/ reports-findings/survreports/mrsa12.pdf

2 Lee BY, Singh A, David MZ, et al. The economic burden of community-associated methicillin-resistant Staphylococcus aureus (CA-MRSA). Clin Microbiol Infect 2013;19:528-36

3 Knapp EA, Salsgiver E, Fink A, et al. Trends in respiratory microbiology of people with cystic fibrosis in the United States, 2006-2012. Ped Pulm 2014; S38:282.

4 CFF Registry CFF. Patient Registry Annual Report. 2013. https://wwwcfforg/2013 CFF_Patient_Registry_Annual_Data_Reportpdf

5 Goss CH, Muhlebach MS. Review: Staphylococcus aureus and MRSA in cystic fibrosis. J Cyst Fibros 2011;10:298-306.

6 Ren $\mathrm{CL}$, Morgan WJ, Konstan MW, et al. Presence of methicillin resistant Staphylococcus aureus in respiratory cultures from cystic fibrosis patients is associated with lower lung function. Pediatr Pulmonol 2007;42:513-18.

7 Muhlebach M, Miller M, Goodrich J, et al. Impact of methicillin resistant $S$. aureus on clinical outcomes in CF. Ped Pulmonology 2007;42:331.

8 Sawicki GS, Rasouliyan L, Ren CL. The impact of MRSA on lung function in patients with cystic fibrosis. Am J Respir Crit Care Med 2009;179:734-5; author reply 35 .

9 Dasenbrook EC, Merlo CA, Diener-West M, et al. Persistent methicillin-resistant Staphylococcus aureus and rate of FEV1 decline in cystic fibrosis. Am J Respir Crit Care Med 2008;178:814-21.

10 Dasenbrook EC, Checkley W, Merlo CA, et al. Association between respiratory tract methicillin-resistant Staphylococcus aureus and survival in cystic fibrosis. JAMA 2010;303:2386-92.

11 Garske LA, Kidd TJ, Gan R, et al. Rifampicin and sodium fusidate reduces the frequency of methicillin-resistant Staphylococcus aureus (MRSA) isolation in adults with cystic fibrosis and chronic MRSA infection. J Hosp Infect 2004;56:208-14.

12 Macfarlane M, Leavy A, McCaughan J, et al. Successful decolonization of methicillin-resistant Staphylococcus aureus in paediatric patients with cystic fibrosis (CF) using a three-step protocol. J Hosp Infect 2007;65:231-6.

13 Solís A, Brown D, Hughes J, et al. Methicillin-resistant Staphylococcus aureus in children with cystic fibrosis: an eradication protocol. Pediatr Pulmonol 2003;36:189-95.

14 Hankinson JL, Odencrantz JR, Fedan KB. Spirometric reference values from a sample of the general U.S. population. Am J Respir Crit Care Med 1999;159:179-87.

15 Wang $X$, Dockery DW, Wypij D, et al. Pulmonary function between 6 and 18 years of age. Pediatr Pulmonol 1993;15:75-88.

16 Buehlmann $M$, Frei $R$, Fenner $L$, et al. Highly effective regimen for decolonization of methicillin-resistant Staphylococcus aureus carriers. Infect Control Hosp Epidemiol 2008:29:510-16.
17 Champion EA, Miller MB, Popowitch EB, et al. Antimicrobial susceptibility and molecular typing of MRSA in cystic fibrosis. Pediatr Pulmonol 2014;49:230-7.

18 Miller MR, Hankinson J, Brusasco V, et al. Standardisation of spirometry. Eur Respir J 2005:26:319-38.

19 Treggiari MM, Retsch-Bogart G, Mayer-Hamblett N, et al. Comparative efficacy and safety of 4 randomized regimens to treat early Pseudomonas aeruginosa infection in children with cystic fibrosis. Arch Pediatr Adolesc Med 2011;165:847-56.

20 Doe SJ, McSorley A, Isalska B, et al. Patient segregation and aggressive antibiotic eradication therapy can control methicillin-resistant Staphylococcus aureus at large cystic fibrosis centres. J Cyst Fibros 2010;9:104-9.

21 Halton K, Zobell J, MacKay R, et al. Evaluation of the effectiveness of a MRSA eradication protocol in pediatric CF patients. Ped Pulm 2009;S32:339 (poster 366)

22 Vanderhelst $\mathrm{E}$, De Wachter $\mathrm{E}$, Willekens J, et al. Increase in ventilated air spaces after eradication of chronic methicillin-resistant Staphylococcus aureus infection in cystic fibrosis patients. Acta Clin Belg 2015;70:30-3.

23 Lo DK, Hurley MN, Muhlebach MS, et al. Interventions for the eradication of meticillin-resistant Staphylococcus aureus (MRSA) in people with cystic fibrosis. Cochrane Database Syst Rev 2015;(2):CD009650.

24 Simor AE, Daneman N. Staphylococcus aureus decolonization as a prevention strategy. Infect Dis Clin North Am 2009;23:133-51.

25 Ridder-Schaphorn S, Ratjen F, Dübbers A, et al. Nasal Staphylococcus aureus carriage is not a risk factor for lower-airway infection in young cystic fibrosis patients. J Clin Microbiol 2007;45:2979-84.

26 West SK, Plantenga MS, Strausbaugh LJ, et al. Use of decolonization to prevent staphylococcal infections in various healthcare settings: results of an Emerging Infections Network survey. Infect Control Hosp Epidemiol 2007;28:1111-13.

27 Muhlebach MS, Miller M, LaVange LM, et al. Treatment intensity and characteristics of MRSA infection in CF. J Cyst Fibrosis 2011:10:201-6.

28 Heltshe SL, Saiman L, Popowitch EB, et al. Outcomes and treatment of chronic methicillin-resistant Staphylococcus aureus differs by Staphylococcal Cassette Chromosome mec (SCCmec) type in children with cystic fibrosis. J Pediatric Infect Dis Soc 2015;4:225-31.

29 Heltshe SL, Goss CH, Thompson V, et al. Short-term and long-term response to pulmonary exacerbation treatment in cystic fibrosis. Thorax 2016;71:223-9.

30 Gibson RL, Emerson J, McNamara S, et al. Significant microbiological effect of inhaled tobramycin in young children with cystic fibrosis. Am J Respir Crit Care Med 2003;167:841-9.

31 Emerson SS. Stopping a clinical trial very early based on unplanned interim analyses: a group sequential approach. Biometrics 1995:51:1152-62.

32 Emerson SS, Banks PLC. Interpretation of a leukemia trial stopped early. In: Lange N, Ryan L, Billiard L, et al, eds. Case studies in biometry. New York City: John Wiley \& Sons, 1994

33 Emerson SS, Fleming TR. Symmetric group sequential test designs. Biometrics 1989:45:905-23.

34 O'Brien PC, Fleming TR. A multiple testing procedure for clinical trials. Biometrics 1979:35:549-56.

35 Pocock SJ. Group sequential methods in the design and analysis of clinical trials. Biometrika 1977:64:191-9. 